\title{
Stable Long-Term Operation of Superconducting Current-Feeder System for the LHD
}

\author{
Shuichi Yamada, Toshiyuki Mito, Ryuji Maekawa, Hirotaka Chikaraishi, Sadatomo Moriuchi, Toshio Uede, \\ Hisaaki Hiue, Yasuo Yasukawa, Ikuo Itoh, Takashi Satow, and Osamu Motojima
}

\begin{abstract}
A superconducting (SC) current-feeder system is used as the current transmission lines for the experimental fusion device, LHD. It consists of nine flexible SC bus lines with total length of $497 \mathrm{~m}$, and nine pairs of gas-cooled current leads. To avoid the propagation of the ice on the leads, the temperature of the terminals had been kept in the range between 5 and 20 degrees Celsius by the heaters. The measured voltage drops of all leads were less than $20 \mathrm{mV}$. The liquid helium levels of the leads and the sub-cooler tank will equalize by the siphon method. The total time of the coil excitations exceeds 3000 hours. We have demonstrated successfully that the SC current-feeder system was stable and easy to handle, and is useful for the SC experimental fusion device.
\end{abstract}

Index Terms-Gas-cooled current leads, LHD, SC bus-line, SC current-feeder system, stable long-term operation.

\section{INTRODUCTION}

$\mathbf{T}$ HE LHD is a large-scale SC toroidal fusion device of heliotron type [1]. One of the main aims of the LHD is to demonstrate the high potentiality of the helical-type device producing current-less steady-state plasma with a sufficiently large Lawson parameter without large current disruption [2]. The main body of the LHD consists of a pair of helical SC coils, three sets of poloidal SC coils and their supporting structure with a total cold mass of 820 tons. Each helical coil is divided into three block coils that can be excited independently. The upper and lower poloidal SC coils are connected in series. Therefore, nine SC bus lines were employed as the current transmission lines for the LHD [3].

The construction of the LHD, including the SC current-feeder system, finished in February of 1998, and the improved plasma confinement properties could be obtained through the first and second experimental cycles in the first year [4]. On the other hand, a partial normal transition was observed in the SC conductor of an inner helical block coil, when engineering coil excitation tests under the high magnetic field regime were conducted. The fault protection programs in the power supplies and the cryogenic system including the SC current-feeder system were successfully executed. To avoid the growth of ice on the current-leads, the mass flow rates of all leads had been decreased to $70 \%$ of the rated values during the nights in the first year [5]. Therefore, it is desirable to realize the operation free and maintenance free system.

Manuscript received September 24, 2001.

S. Yamada, T. Mito, R. Maekawa, H. Chikaraishi, S. Moriuchi, T. Satow, and O. Motojima are with the National Institute for Fusion Science, 322-6 Oroshi, Toki, Gifu 509-5292, Japan (e-mail: yamadas@LHD.nifs.ac.jp).

T. Uede, H. Hiue, Y. Yasukawa, and I. Itoh are with the Fuji Electric Co., Ltd., Kawasaki, Kanagawa, 210-8530, Japan.

Publisher Item Identifier S 1051-8223(02)04190-8.
TABLE I

COMPARISON OF MERIT AND DEMERIT FOR USING THE FleXIBLE SC BuS LINE AND WATER COOLED (WC) BUS-BAR

\begin{tabular}{lcc}
\hline Item & SC bus-line & WC bus-line \\
\hline Manufacturing costs & $\bigcirc$ & $\bigcirc$ \\
Running costs & $\bigcirc$ & $\triangle$ \\
Space around the main device & ( & $\triangle$ \\
Assembling works on site & $\bigcirc$ & $\triangle$ \\
Capacity of the power supply & $\bigcirc$ & $\triangle$ \\
Handling & $\bigcirc$ & $\bigcirc$ \\
Maintenance & $\bigcirc$ & $\bigcirc$ \\
Safety & $\bigcirc$ & $\bigcirc$ \\
Reliabilities & $\bigcirc$ & $\bigcirc$ \\
\hline
\end{tabular}

(): excellent, $\bigcirc$ : good, $\triangle$ : Average

TABLE II

MAIN PARAMETERS OF AN SC BUS-LINE FOR THE LHD

\begin{tabular}{ll}
\hline Items & Specifications \\
\hline Number & Nine \\
$\quad$ (for helical coils) & (Six) \\
(for poloidal coils) & (Thre) \\
Total length of $9 \mathrm{SC}$ bus-lines & $497 \mathrm{~m}$ \\
Rated current & $\mathrm{dc} 32 \mathrm{kA}$ \\
Withstand Voltage & $\mathrm{dc} 5 \mathrm{kV} @ 77 \mathrm{~K}$ \\
Diameter of bus line & $230 \mathrm{~mm}$ \\
Minimum bending radius & $1.5 \mathrm{~m}$ \\
Cryogenic transfer-line & five corrugated SUS tubes \\
Mass flow rate of two-phase helium & $12 \mathrm{~g} / \mathrm{s}$ (max. value) \\
\hline
\end{tabular}

This paper describes a trial for the automatic manipulation for the SC current-feeder system of the LHD. The overall heat balance adjustments of the SC current-feeder system is also discussed.

\section{SC CURRENT FEEDER SYSTEM}

The application of the SC bus lines leads to a decrease of the electrical power consumption for the power supplies. Space for the heating devices and/or diagnostics apparatus around the LHD enable to expand, by locating the current leads apart from it. The SC bus lines of the LHD have flexibility, since the route from the SC coils to their power supplies have many corners and bends to avoid the direct shine-through of the neutron. The merits for using the SC bus lines are summarized in Table I.

The SC bus line has a sufficient safety margin exceeding that of the SC coils of the LHD. It maintains a rated current carrying capacity for 30 minutes, even if the coolants supplied to the SC bus line are accidentally stopped [6]. The minimum bending radius is determined to be $1.5 \mathrm{~m}$, because of the installation routes. The main parameters of the SC bus lines are listed in Table II. 
TABLE III

SPECIFICATIONS OF THE CURRENT LEAD

\begin{tabular}{lccc}
\hline Target coil & Helical coil & IV, IS coil & OV coil \\
\hline Number of leads & 6 pairs & 2 pairs & 1 pair \\
Rated current & $18 \mathrm{kA}$ & $22 \mathrm{kA}$ & $32 \mathrm{kA}$ \\
Rated mass flow & $1.08 \mathrm{~g} / \mathrm{s}$ & $1.3 \mathrm{~g} / \mathrm{s}$ & $1.9 \mathrm{~g} / \mathrm{s}$ \\
(without current case) & $(0.8 \mathrm{~g} / \mathrm{s})$ & $(0.9 \mathrm{~g} / \mathrm{s})$ & $(1.4 \mathrm{~g} / \mathrm{s})$ \\
Length & $1.5 \mathrm{~m}$ & $1.5 \mathrm{~m}$ & $1.5 \mathrm{~m}$ \\
\hline
\end{tabular}

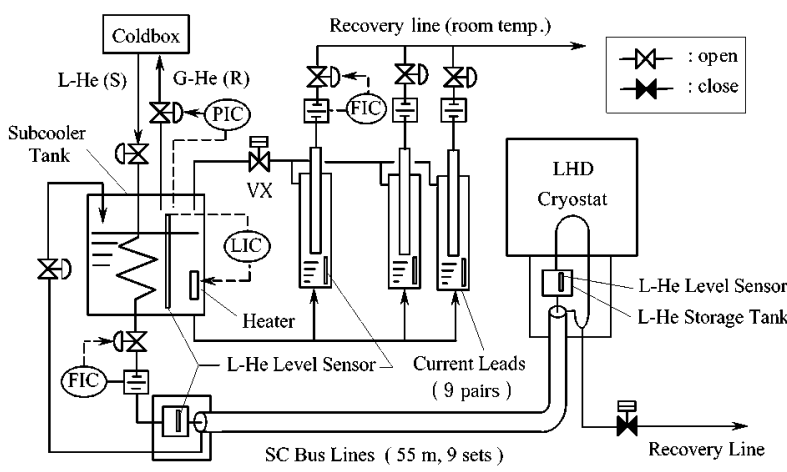

Fig. 1. Control scheme of the helium flow in the SC current-feeder system.

The SC current-feeder system is composed of two sub-cooler tanks, nine pairs of current leads and nine sets of SC bus lines, including the cryogenic valves and their controller. The coolants of the SC current-feeder system are separated from that of the SC coil system of the LHD.

An aluminum-stabilized, SC-compacted stranded cable that is the same size and same structure of the full-scale model, was applied to the SC bus lines for the LHD. A pair of SC cables was insulated electrically, and inserted into the cryogenic transfer line. To decrease the heat load into the SC bus lines, five-corrugated stainless-steel tubes with a thermal shielding channel were used. Each process of a cooling-down, steady state and warming-up are controlled by the sequential program that is linked together with the program for the helium refrigerator/liquefier system.

The conventional gas-cooled current leads with the helium storage were applied to attain the stable current carrying properties at a rated current. The normalized heat load of these current leads at $4 \mathrm{~K}$ level was designed to be $1.2 \mathrm{~W} / \mathrm{kA}$. Main parameters of the current leads are listed in Table III.

Fig. 1 illustrates a control scheme of a helium flow for the SC current-feeder system. In a steady-state, two-phase helium from the coldbox is cooled to $4.4 \mathrm{~K}$ by the heat exchanger in the subcooler tank, and is supplied to the SC bus lines. Returned twophase helium from the $\mathrm{SC}$ bus line flows into the sub-cooler, and separates into liquid and gas phase. A part of the liquid helium cools the current leads. Most of the liquid helium evaporates by the heaters, and returns to the coldbox. The PID compensators for the cryogenic valves and the heaters adjust automatically for the manipulated values of the pressures in the sub-coolers, and mass flow rates of the SC bus lines and current leads, and the liquid helium levels in the sub-coolers [7].

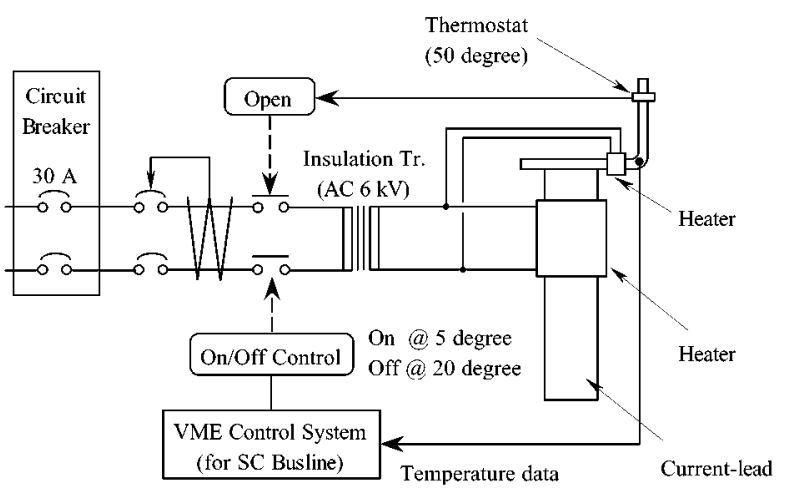

Fig. 2. Electrical circuit diagram of the heaters on the current-leads.

TABLE IV

OPERATIONS FOR CURRENT-LEAD IN LHD

\begin{tabular}{lcc}
\hline $\begin{array}{c}\text { Experimental Cycles } \\
\text { in LHD }\end{array}$ & $\begin{array}{c}\text { Temp. control for } \\
\text { the terminals }\end{array}$ & $\begin{array}{c}\text { Mss flow Adjustments for } \\
\text { current carrying rate }\end{array}$ \\
\hline 1st Cycle (1998FY) & No & Every time \\
2nd Cycle (1998FY) & No & Every time \\
3rd Cycle (1999FY) & $5-20^{\circ} \mathrm{C}$ & Automatic control \\
4th Cycle (2000FY) & $5-20^{\circ} \mathrm{C}$ & Automatic control \\
5th Cycle (2001FY) & $5-20^{\circ} \mathrm{C}$ & Automatic control \\
\hline
\end{tabular}

\section{Temperature Control of the CURRENT LeAD}

The temperature of the warm-end terminals of the current lead depends on the heat balance between the cooling condition and heat inputs such as thermal conduction from the bus-bar and the heat transfer from the air. When the ice covers on the terminal of the current lead, its may gradually grow to the large ice block, because the ice will act like an adiabatic materials. To suppress the growth of ices on the leads, the mass flow rates of all leads had been decreased to $70 \%$ of the rated values during nights, and the ices had been extracted on every weekend in the first year [5].

To release these operation and maintenance for the ices on the leads, two electrical heaters were attached on the terminal of all current leads before starting the third experimental cycle in 1999 FY. The electrical circuit diagram of the heaters on the current leads is shown in Fig. 2. The current leads and power circuits for the heaters was isolated by the transformer with ac $6 \mathrm{kV}$ withstand voltage. The history of the operations for the current leads in LHD is summarized in Table IV.

Fig. 3 shows the overall characteristics of the current leads by using the heater control; (a) currents, (b) voltage drop of the leads, (c) temperatures of the warm-end terminals, and (d) on/off states of the heaters. The temperature of the terminals has been monitored continuously, and kept in the range between 5 and 20 degrees Celsius by the on/off control of the heater circuits. One cycle from on-state to off-states of the heater, depends on the current carrying rate in the lead, the power of the heater and/or temperature difference of on/off states, is tuned to the order of one hour. 
(a)

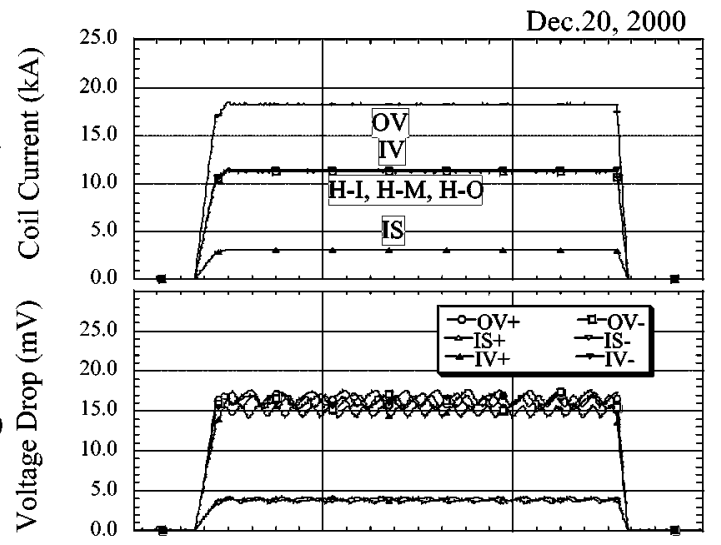

(c)

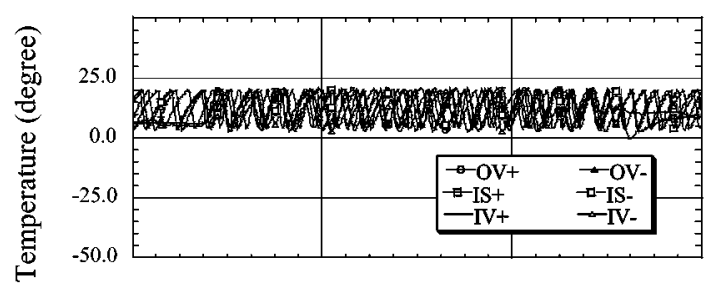

(d)

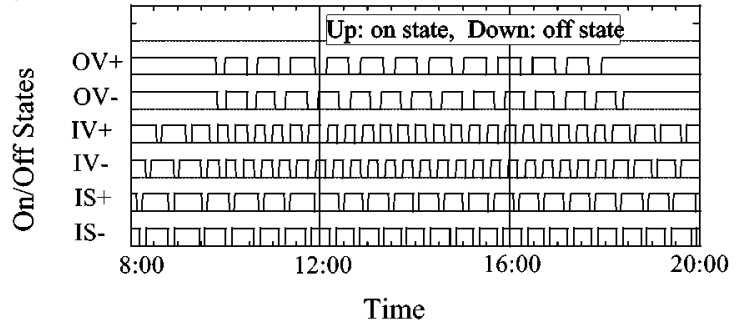

Fig. 3. Time evolution of coil currents, temperatures and voltage drops of the leads, and on/off states of the heaters.

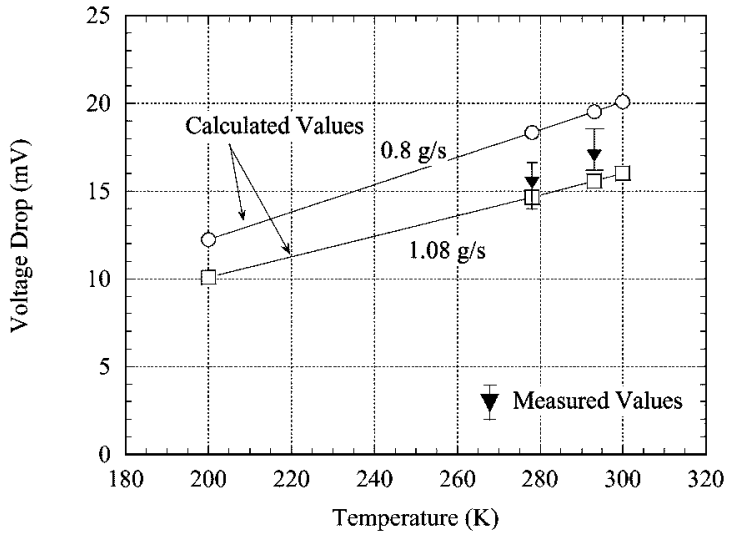

Fig. 4. Measured and calculated voltage drop of the leads as a function of the temperature of the warm-end terminal.

The voltage drop of the lead also depends strongly on the temperature of the warm-end terminal.

Fig. 4 illustrates the measured voltages drop of the lead as a function of the temperature of the warm-end terminal in comparison with that of calculated one. The calculation was conducted by solving the one-dimensional energy balance between the cooling term by the coolant, and heating terms by (a)

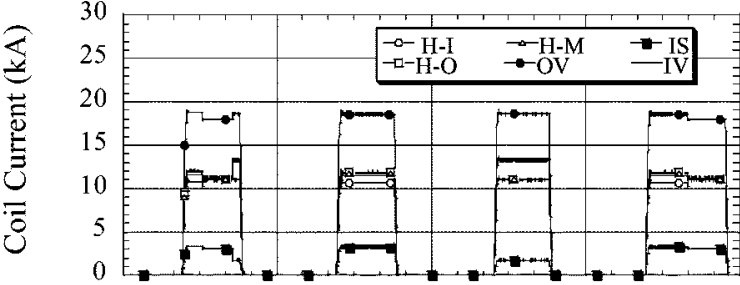

(b)

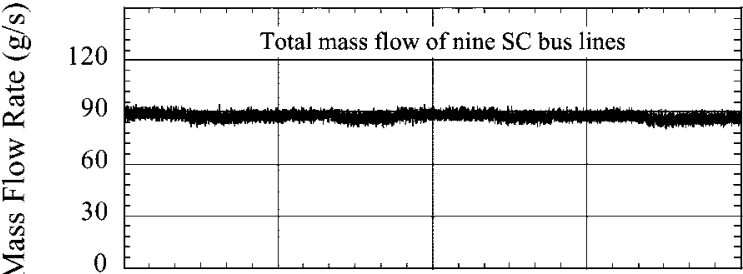

(c)

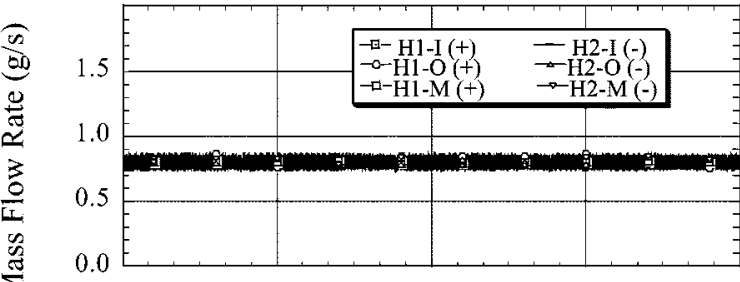

(d)

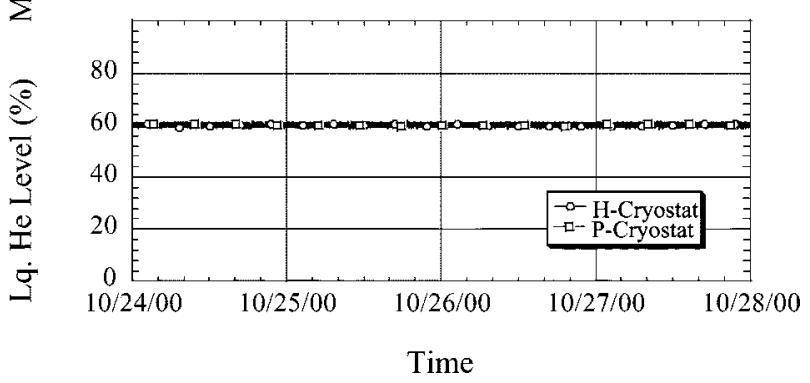

Fig. 5. Typical example of the steady-state operation: (a) coil currents, (b) total mass flow rate of nine SC bus-lines, (c) mass flow rate of the current-leads for helical coil, and (d) liquid helium level in the sub-coolers.

the thermal conduction and Joule heat of the conductor [8]. As shown Table III, the mass flow rates of 1.08 and $0.8 \mathrm{~g} / \mathrm{s}$ are designed values of the lead for helical coil system. It is confirmed that measured voltage drop of the lead shows good agreements with that of the calculated value. The voltage drops of less than $20 \mathrm{mV}$ have a large margin for the safety operation of the leads.

\section{STEADY-STATE OPERATION}

Fig. 5 gives a typical example of the steady-state operation; (a) coil current, (b) total mass flow rate of nine SC bus lines, (c) mass flow rate of the current leads for helical coils, and (d) liquid helium levels in the sub-coolers. The SC coils of the LHD produce equilibrium magnetic field without plasma current, so that they are continuously charged up for nine or twelve hours in a daytime. Total mass flow rate of two phase helium of nine SC bus lines had been kept in constant value of $90 \mathrm{~g} / \mathrm{s}$. In generally, mass flow rate of the current lead should be adjusted to the suitable value in response to the current carrying rate. However, the mass flow rates in this system have been kept in constant value, even if the coil current changes from zero to the nominal value of $3 \mathrm{~T}$ operation.

Fig. 6 shows a typical example of the automatic heat-balance adjustment; (a) heater powers in the sub-coolers, (b) pressures 
(a)

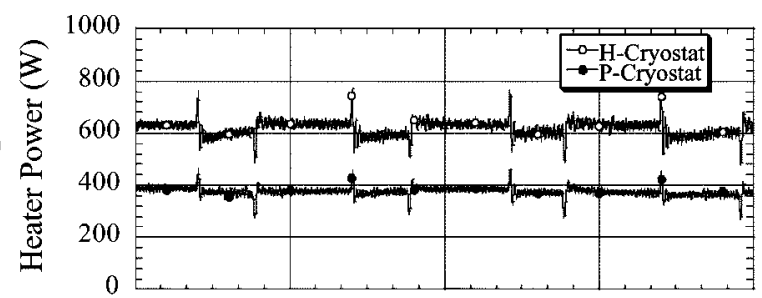

(b)

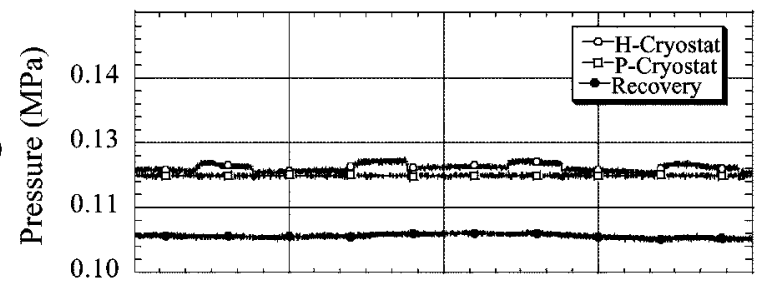

(c)

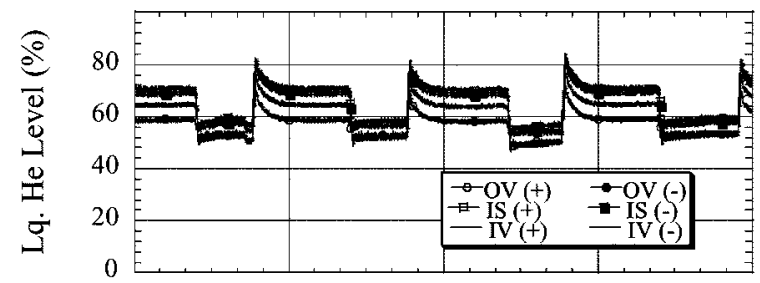

(d)

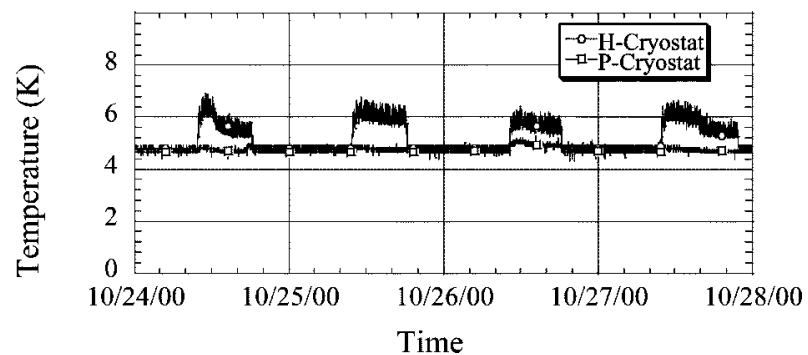

Fig. 6. Typical example of the steady-state operation: (a) heater power and (b) pressure in the sub-cooler, (c) liquid helium in the leads, and (d) temperature of helium gas returned to the coldbox.

TABLE V

Main Parameter of THE STEADY-STATE Operation

\begin{tabular}{ll}
\hline Parameters & Values \\
\hline SC Bus-Line & \\
Mass flow rate of L-He & $8.0-12.0 \mathrm{~g} / \mathrm{s}$ \\
Mass flow rate of shielding G-He & $1.8-2.2 \mathrm{~g} / \mathrm{s}$ \\
Temperature of two-phase helium & $4.4-4.5 \mathrm{~K}$ \\
Temperature of shielding G-He & 51 (in) -69 (out) K \\
Sub-Coolers & \\
Pressures & $0.115-0.118 \mathrm{MPa}$ \\
Liquid helium levels & $60 \%$ \\
Heater power (for Helical cryostat) & $500-800 \mathrm{~W}$ \\
$\quad$ (for poloidal cryostat) & $300-500 \mathrm{~W}$ \\
Others parameter & \\
Temperature of helium gas to coldbox & $4.3-7 \mathrm{~K}$ \\
Pressure of recovery line & $0.104-0.106 \mathrm{MPa}$ \\
\hline
\end{tabular}

in the sub-coolers and recovery line, (c) liquid helium levels in the leads, and (d) temperature of the helium gas returned to the coldbox. The mass flow rate and current carrying rate influence the cooling conditions of the current leads. However, both liquid helium levels in the sub-cooler and current leads will automatically equalize by a siphon method without the mass-flow adjustment in the leads, when the valve VX shown in Fig. 1
TABLE VI

SUMMARY OF THE EXPERIMENTAL CYCLE OF THE LHD

\begin{tabular}{lcc}
\hline $\begin{array}{c}\text { Experimental Cycles } \\
\text { in LHD }\end{array}$ & $\begin{array}{c}\text { Plasma shots } \\
\text { numbers }\end{array}$ & $\begin{array}{c}\text { Times of coil } \\
\text { excitations }\end{array}$ \\
\hline 1st Cycle (1998FY) & $\mathbf{1 8 8 8 \text { shots }}$ & 692 hours \\
2nd Cycle (1998FY) & 5244 shots & 1134 hours \\
3rd Cycle (1999FY) & 10191 shots & $\mathbf{6 7 9}$ hours \\
4th Cycle (2000FY) & $\mathbf{8 8 9 6}$ shots & $\mathbf{6 0 9}$ hours \\
\hline
\end{tabular}

was open. Even if the mass-flow rates of the current leads were less than that of the self-cooling condition, a part of the evaporated helium gas from the current leads flowed into the subcooler through the valve VX, and returned to the coldbox. The Joule heats in the leads are automatically absorbed by the small changes of the heater powers in the sub-cooler, and the pressure and temperature of the helium gas in the sub-cooler, during the coil excitation phase. In steady-state, the heaters automatically adjust the overall heat balance between the SC current-feeder system and the refrigerator/liquefier system. Main parameters of steady-state operations are listed in Table V.

Table VI summarizes the experimental cycles in LHD. Total shot number of plasma production were 26208 , and total time of coil excitations exceeds 3000 hours. We have demonstrated successfully that the SC current-feeder system was stable and easy to handle, and was useful for the SC experimental fusion device.

\section{CONCLUSIONS}

The overall operational characteristics of the SC current-feeder system for the LHD were investigated. The results are concluded as follows: 1) The temperature of the terminals had been kept in the range between 5 and 20 degrees Celsius by the heaters. 2) The measured voltage drops of all leads are less than $20 \mathrm{mV}$. 3) Mass-flow rates, pressures and liquid-helium levels of the SC bus lines and current leads are well controlled automatically in steady-state operation. 4) The total time of the coil excitations exceeds 3000 hours. We have demonstrated successfully that the SC current-feeder system with high-current capacities was reliable and safe, and was useful for the SC experimental fusion device.

\section{REFERENCES}

[1] A. Iiyoshi et al., "Overview of the large helical device," Nuclear Fusion, vol. 39, pp. 1245-1256, 1999.

[2] M. Fujiwara et al., "Overview of long pulse operation in the large helical device," Nuclear Fusion, vol. 40, pp. 1157-1166, 2000.

[3] T. Mito et al., "Development and tests of a flexible superconducting bus-line for the large helical device," IEEE Trans. Magn., vol. 30, pp. 2090-2093, 1994

[4] O. Motojima et al., "Progress summary of LHD engineering design and construction," Nuclear Fusion, vol. 40, pp. 599-609, 2000.

[5] S. Yamada et al., "Overall operating characteristics of superconducting current-feeder system for the LHD," Advances in Cryogenic Engineering, vol. 45, pp. 1525-1532, 2000.

[6] S. Yamada et al., "Superconducting current feeder system for the large helical device," IEEE Trans. Magn., vol. 32, pp. 2422-2425, 1996.

[7] S. Yamada et al., "First cycle operation of SC current feeder system for the LHD," in Proc. 17th ICEC, Bournemouth, UK, 1998, pp. 443-446.

[8] E. Tada et al., "Development of high-current vapor-cooled current lead for fusion devices," Advances in Cryogenic Engineering, vol. 31, pp. 225-233, 1986. 